\title{
Self-monitoring of urinary salt excretion as a method of salt-reduction education: a parallel, randomized trial involving two groups
}

\author{
Kenichiro Yasutake ${ }^{1, *}$, Emiko Miyoshi ${ }^{1}$, Yukiko Misumi ${ }^{2}$, Tomomi Kajiyama ${ }^{1}$, \\ Tamami Fukuda $^{3}$, Taeko Ishii ${ }^{4}$, Ririko Moriguchi ${ }^{1}$, Yusuke Murata ${ }^{5}$, Kenji Ohe ${ }^{5}$, \\ Munechika Enjoji ${ }^{5}$ and Takuya Tsuchihashi ${ }^{6}$ \\ 'Department of Nutritional Sciences, Faculty of Nutritional Sciences, Nakamura Gakuen University, 5-7-1 Befu, \\ Jonan-ku, Fukuoka 814-0198, Japan: ${ }^{2}$ Department of Health and Nutrition Sciences, Faculty of Health and Nutrition \\ Sciences, Nishikyushu University, Saga, Japan: ${ }^{3}$ Fukuoka Clinic, TOPPAN Group Health Insurance Society, Fukuoka, \\ Japan: ${ }^{4}$ Department of Food Design, Kurume Shin-ai Women's College, Kurume, Japan: ${ }^{5}$ Health Care Center, \\ Fukuoka University, Fukuoka, Japan: ${ }^{6}$ Hypertension Center, Steel Memorial Yawata Hospital, Kitakyushu, Japan
}

Submitted 11 September 2017: Final revision received 10 December 2017: Accepted 21 December 2017: First published online 20 February 2018

\begin{abstract}
Objective: The present study aimed to evaluate salt-reduction education using a self-monitoring urinary salt-excretion device.

Design: Parallel, randomized trial involving two groups. The following parameters were checked at baseline and endline of the intervention: salt check sheet, eating behaviour questionnaire, $24 \mathrm{~h}$ home urine collection, blood pressure before and after urine collection.

Setting: The intervention group self-monitored urine salt excretion using a selfmeasuring device for 4 weeks. In the control group, urine salt excretion was measured, but the individuals were not informed of the result.

Subjects: Seventy-eight individuals (control group, $n$ 36; intervention group, $n$ 42) collected two $24 \mathrm{~h}$ urine samples from a target population of 123 local resident volunteers. The samples were then analysed.

Results: There were no differences in clinical background or related parameters between the two groups. The $24 \mathrm{~h}$ urinary $\mathrm{Na}: \mathrm{K}$ ratio showed a significant decrease in the intervention group $(-1 \cdot 1)$ compared with the control group $(-0 \cdot 0$; $P=0.033$ ). Blood pressure did not change in either group. The results of the salt check sheet did not change in the control group but were significantly lower in the intervention group. The score of the eating behaviour questionnaire did not change in the control group, but the intervention group showed a significant increase in eating behaviour stage.

Conclusions: Self-monitoring of urinary salt excretion helps to improve $24 \mathrm{~h}$ urinary Na:K, salt check sheet scores and stage of eating behaviour. Thus, usage of self-monitoring tools has an educational potential in salt intake reduction.
\end{abstract}

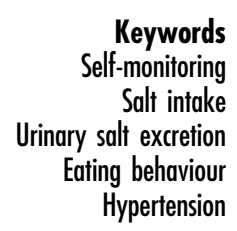

Excessive salt intake increases blood pressure with the eventual outcome of stroke and CVD, making it the second most important risk factor to harm one's health next to cigarette smoking ${ }^{(1,2)}$. In fact, $10 \%$ of cardiovascular deaths worldwide are said to be caused by excessive salt intake $^{(3)}$. Conversely, continuous reduction in salt intake mitigates these risks ${ }^{(4)}$.

Both developed and developing countries have reported excessive salt intake in their populations ${ }^{(5,6)}$, although the sources of salt vary. In Japan, exceptionally high salt intake is an important lifestyle issue ${ }^{(6,7)}$. Despite this, no effective salt-reduction education has yet been established.

Several society-wide approaches, such as salt reduction in processed foods or clear nutritional labelling, have been effective $^{(8)}$. However, these necessitate cooperation between government and industry. In contrast, individual approaches, such as salt-reduction education, make people aware of their own salt intake. For example, $24 \mathrm{~h}$ urinary salt excretion analysis provides individuals with feedback regarding their salt levels, leading to lower salt intake in the short and long terms ${ }^{(9,10)}$. 
However, it is not feasible for people to repeatedly conduct $24 \mathrm{~h}$ urine collections, and spot-urine or secondurine tests, which are performed immediately after waking, must be measured by an inspection agency, so they are impractical for individuals ${ }^{(11,12)}$. Alternatively, $24 \mathrm{~h}$ urinary salt excretion can be estimated based on overnight (ON) salt excretion levels using a self-monitoring device (KME-03; Kohno ME Laboratory, Kanagawa, Japan). Individuals can do this themselves and the method shows significant correlation with the $24 \mathrm{~h}$ urine collection method ${ }^{(13)}$. Furthermore, the urinary salt 'in-out' method - which employs a self-monitoring urinary salt-excretion device has been compared with $24 \mathrm{~h}$ urinary excretion collection. The in-out method showed significant accuracy and could be used to motivate participants ${ }^{(14-16)}$. Moreover, individuals who used the self-monitoring urinary salt-excretion device showed effective management of their salt intake despite receiving no education on how to reduce their short-term ON urinary salt excretion levels ${ }^{(17)}$. Nevertheless, no allocation tests, such as randomized comparison tests, have yet been conducted to evaluate the effectiveness of the device.

The present study was a parallel, randomized trial involving two groups; it aimed to evaluate whether the self-monitoring urinary salt-excretion device reduces salt intake over 4 weeks.

\section{Methods}

\section{Participants}

The target population comprised 123 out of 162 individuals who responded to a poster campaign and subsequent explanatory meeting that took place between September and November 2014. The posters were displayed on noticeboards in regional clusters of about 1300 apartment buildings, three health-care centres and one company. All individuals agreed to participate by both verbal and written agreement. Among the recruited individuals, those with a history of hypertension or antihypertensive drug use other than diuretics were not excluded. The research poster and explanatory meeting made it clear that individuals would not be eligible to participate if they: (i) had physical, mental or social difficulties; (ii) took diuretics; (iii) were pregnant; (iv) had oedema or renal disease; or (v) could not make decisions for themselves. In addition, only participants above 21 years of age were recruited. Individuals who answered these criteria were excluded from the study.

\section{Intervention scbedule}

Upon entry into the study, the participants were asked to answer the salt check sheet ${ }^{(18)}$ and a questionnaire on eating behaviour that was modified using the transtheoretical model (Fig. 1) ${ }^{(19)}$. They were also asked to collect and submit a $24 \mathrm{~h}$ urine sample on a convenient day within 2 weeks of entry. Women were asked to collect urine on a day outside the menstrual period. At this time, they were instructed to continue their typical dietary habits. After urine collection, the participants were asked to measure their blood pressure.

This initial collection provided the baseline values for gender, age, existence of hypertension, blood pressure and $24 \mathrm{~h}$ urinary salt excretion. These were used as key parameters to stratify randomization and divide the subject population equally into two groups: an intervention group that used the self-monitoring urinary salt-excretion device and a control group that did not. The intervention group was asked to use the self-monitoring urinary salt-excretion device for 4 weeks and plot the measurements on a graph sheet (see below). At the end of the investigation, they were asked to record the results of their $24 \mathrm{~h}$ home urine collection, blood pressure measurement, salt check sheet and eating behaviour questionnaire (endline investigation). Conversely, the control group was asked to act in a manner typical to their everyday life from the time of entry. After 4 weeks, they answered the salt check sheet and eating behaviour questionnaire (endline investigation). Neither group received any nutritional education. The results of the $24 \mathrm{~h}$ urinary salt excretion were recorded individually after the study ended.

The 4-week duration of the study was based on our own pilot studies, wherein ON salt excretion levels decreased after 3-4 weeks of using the self-monitoring urinary salt-excretion device ${ }^{(17)}$. Moreover, 4 weeks was long enough to confirm the antihypertensive effect, as we have shown in our previous study ${ }^{(15,20)}$.

\section{Outcomes}

The primary outcomes of the present study were: (i) the difference in $24 \mathrm{~h}$ urinary salt excretion after the intervention; and (ii) the difference between the intervention and control groups. Since salt intake differs greatly between individuals ${ }^{(21)}, 24 \mathrm{~h}$ urinary collection should be performed on multiple days ${ }^{(22)}$. However, the compliance rate decreases after multiple iterations ${ }^{(16)}$, resulting in a low number of samples available for analysis. Thus, we instructed the participants to collect $24 \mathrm{~h}$ urine samples only at baseline and endline in the present study.

The secondary outcomes were change in the $\mathrm{Na}: \mathrm{K}$ ratio and $\mathrm{ON}$ salt excretion in the $24 \mathrm{~h}$ urine sample, as well as blood pressure.

\section{Twenty-four-bour urine collection}

A partition cup (proportional sampling method) was used for the $24 \mathrm{~h}$ home urine collection and 1/50 of the total urine volume was collected ${ }^{(23)}$. Reports comparing the partition cup and total urine method show a high correlation index of urine volume $(r=0.97)$ and urinary salt excretion $(r=0.98)^{(23)}$. To collect urine accurately using the partition cup, the participants were given verbal and written instructions, as well as a simulation, beforehand. 


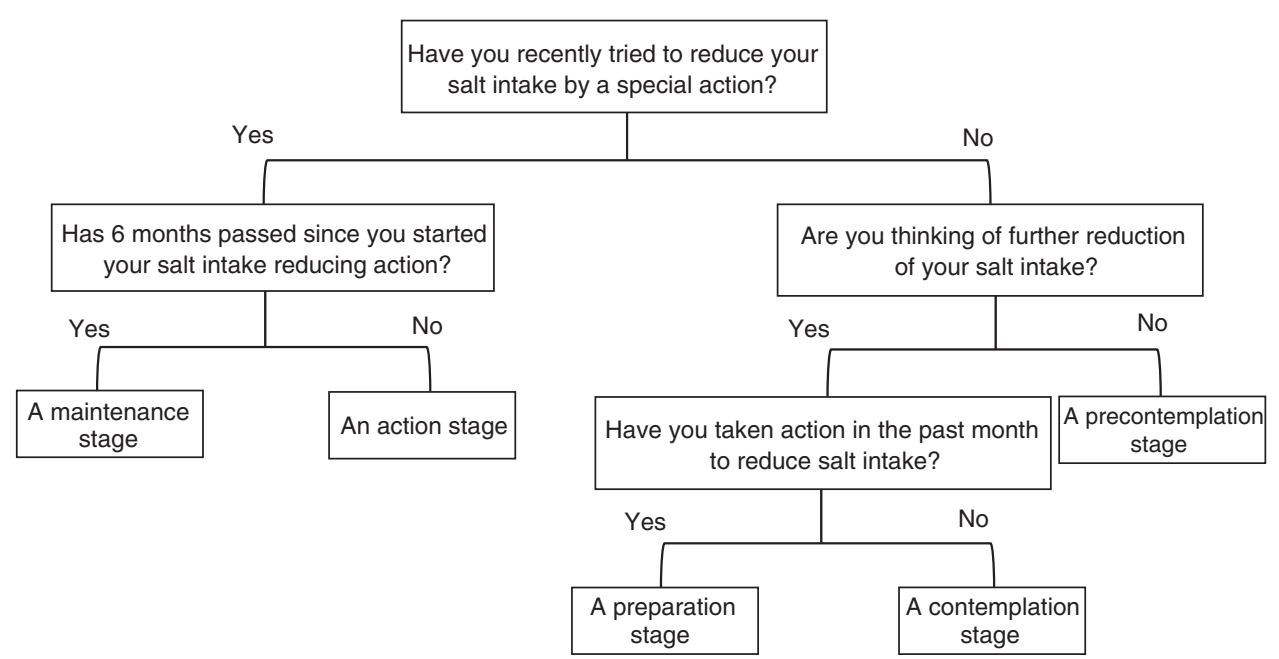

Fig. 1 Questionnaire about salt-reduction eating behaviour stage. The list was based on the transtheoretical model

The participants were specifically reminded to collect urine during defecation.

To empty their bladders after the first-morning urination, the participants were asked to complete urination at 06.00 hours, which is the standard urine collection time. Thereafter, the participants were asked to collect all urine using the partition cup. Finally, they were asked to complete another scheduled urination at 06.00 hours the next day, regardless of desire to urinate. The total volume up to then was designated as the $24 \mathrm{~h}$ home urine collection ${ }^{(15,16,24,25)}$. The participants' urine samples were collected occasionally and analysed. SRL Co., Ltd analysed the total urine volume, as well as urine creatinine, $\mathrm{Na}$ and $\mathrm{K}$ levels. Specifically, creatinine was analysed by enzyme assay, $\mathrm{Na}$ and $\mathrm{K}$ by the electrode method. Urine samples whose measured $24 \mathrm{~h}$ creatinine levels were within $30 \%$ of the estimated levels were designated as successful. Those outside this range were eliminated ${ }^{(15,16,24,25)}$.

\section{Self-monitoring device}

A self-monitoring device (KME-03; Kohno ME Laboratory, Kanagawa, Japan) was used in the present study. The device can estimate $24 \mathrm{~h}$ salt excretion from $8 \mathrm{~h}$ ON urine samples in an exclusive container. Therefore, participants were asked to urinate $8 \mathrm{~h}$ before their prearranged waking time (06.00 hours). Urine evacuated during the sleeping period was collected in a dedicated cup. If an unscheduled urination was needed between 22.00 and 06.00 hours, the urine was added to the first-morning urine and analysed.

The principle of the measuring device is as follows: the volume sensor consists of fifty small, resistant chips and a conductivity sensor comprising two gold-coated nickel plates. These sensors measure urine volume, $\mathrm{Cl}^{-}$ concentration and temperature. These values are then integrated, and the $\mathrm{NaCl}$ concentration is estimated using an original algorithm. In the present study, because conductivity-based concentration measurement is affected by other electrolytes, such as $\mathrm{K}$, the $\mathrm{NaCl}$ concentration was adjusted using a correlation formula so that it was between the values obtained using the ion electrode and conductivity methods. The self-monitoring device estimated $24 \mathrm{~h}$ salt excretion from $\mathrm{ON}$ urine samples using the following formula: $Y=5.76 X \times 0.53(\mathrm{~g} / \mathrm{d})$, where $Y$ is the estimated $24 \mathrm{~h}$ urinary salt excretion and $X$ is the $\mathrm{Na}^{+}$ content of the ON urine samples ${ }^{(13)}$. This formula was derived from regression analysis of $24 \mathrm{~h}$ salt excretion and of those estimated using the self-monitoring device to analyse $\mathrm{ON}$ urine samples. Moreover, others have reported that $24 \mathrm{~h}$ salt excretion correlates well with ON urinary salt excretion $(r=0.72, P<0.001 ; n 224)^{(13)}$. We have also confirmed the validity of the self-monitoring device in previous studies ${ }^{(14-16)}$. First, test meals containing known amounts of salt $(5,10$ and $13 \mathrm{~g} / \mathrm{d})$ confirmed that overnight urinary salt excretion values roughly reflect the previous day's salt intake, but correlation of $\mathrm{ON}$ to $24 \mathrm{~h}$ urinary salt excretion was found only in the $10 \mathrm{~g}$ salt/d condition ${ }^{(14,15)}$. Second, we have reported CV for interday differences for overnight urinary salt excretion. The CV for $24 \mathrm{~h}$ urinary salt excretion among three test days and among subjects were 24.7 and $21.3 \%$, respectively. The CV for overnight urinary salt excretion were lower than those for $24 \mathrm{~h}$ salt $(13.3$ and $17 \cdot 7 \%$, respectively) ${ }^{(16)}$.

\section{Recording overnight salt excretion level using the self-monitoring urinary salt-excretion device}

The participants were asked to record their urinary salt excretion levels every morning using the device and plot them on a graph sheet. The graph was prepared by modifying the bodyweight graph diary developed for obese patients ${ }^{(26)}$. The participants were asked to draw a red line at the target value of salt intake (males, below $8 \mathrm{~g} / \mathrm{d}$; females, below $7 \mathrm{~g} / \mathrm{d}$; hypertensive people, below $6 \mathrm{~g} / \mathrm{d})^{(27,28)}$ detailed in the Japanese dietary salt intake standard (2015). They were then asked to connect these values to their everyday diet and try to lower their values near to the target. 


\section{Salt check sheet}

The salt check sheet is a single A4 sheet with thirteen questions; it takes $3-5 \mathrm{~min}$ to complete. The thirteen questions can be categorized as follows: seven items evaluate the intake of salty meals, such as miso soup, pickles and noodles; four evaluate the use of salty sauces (e.g. soya sauce), eating out and home-meal replacement; and two evaluate the seasoning content and size of homemade meals. Each question is scored up to 3 points, with a total of 35 points, and salt content is characterized as low (0-8 points), medium (9-13 points), high (14-19 points) or very high $\left(\geq 20\right.$ points) ${ }^{(18)}$. The check sheet was based on the relationship between salt intake (estimated using a brief self-administered diet history questionnaire $)^{(29,30)}$ and $24 \mathrm{~h}$ urinary salt excretion in outpatients with hypertension in Fukuoka prefecture ${ }^{(31)}$. The validity of the values from the salt check sheet were checked in studies involving patients with hypertension and local middle-aged people ${ }^{(18,24)}$.

\section{Questionnaire about salt-reducing eating behaviour}

Generally, desirable health behaviour is attained in five stages, as evaluated by the transtheoretical model: (i) a precontemplation stage, in which a patient has no intention of changing in the foreseeable future; (ii) a contemplation stage, in which a patient intends to change, but not soon; (iii) a preparation stage, in which a patient intends to change during the next month; (iv) an action stage, in which a patient changes; and (v) a maintenance stage, in which a patient maintains the change for at least 6 months $^{(19)}$. We referred to a previous report in which the transtheoretical model showed that spot-urine measurement is comparable with $24 \mathrm{~h}$ urinary salt excretion ${ }^{(32)}$. The same study categorized the eating behaviour of each individual into five stages using a questionnaire (Fig. 1).

\section{Blood pressure measurement}

The participants measured their blood pressure, after resting for $5 \mathrm{~min}$, using a digital, upper-arm, automatic blood pressure-measuring instrument (HEM-7080 IC; Omron, Kyoto, Japan). They did so at the beginning and end of the $24 \mathrm{~h}$ urine collection period - immediately after the early-morning urination. The mean of four consecutive measurements was recorded.

\section{Antbropometric measurements}

Fasting measurements of height and body weight were conducted after urine sampling on the last day of $24 \mathrm{~h}$ urine collection, with the participants lightly dressed and without shoes or socks. Their body weight was self-measured at home using the same scale at baseline and endline. Their height was self-declared, and body weight measurements were recorded to the nearest $0 \cdot 1 \mathrm{~kg}$. BMI was calculated using these values of height and body weight.

\section{Quality assurance of data collection}

The authors pre-practised all investigations, including the $24 \mathrm{~h}$ urine collection, to become familiar with the procedure, data collection and confirmation; and could therefore adequately explain these processes. Blanks or errors in the salt check sheet ${ }^{(18)}$ or questionnaire about salt-reducing eating behaviour ${ }^{(19)}$ were corrected by asking each individual.

\section{Statistical analysis}

Statistical data were presented as means and standard deviations. The $\chi^{2}$ test was used to analyse qualitative variables, the paired $t$ test was used to compare two paired groups, and the unpaired $t$ test to compare two unpaired groups. Concerning urinary salt excretion and blood pressure, the difference between baseline and endline was corrected as a covariate using ANCOVA. One-way ANOVA was used when there were three or more groups. The significance level was set at $P<0 \cdot 05$. The statistical software package IBM SPSS Statistics version 22 was used in the statistical analyses.

\section{Results}

The present study involved 123 participants, who were assigned by stratified randomization: sixty-two in the control group and sixty-one in the intervention group. Six individuals withdrew their consent during the study: two in the control group and four in the intervention group. Seventy-eight participants succeeded in collecting a $24 \mathrm{~h}$ home urine sample at both baseline and endline: thirty-six in the control group and forty-two in the intervention group (baseline and endline). These samples were subjected to analyses (Fig. 2). Ultimately, the participants comprised twelve men and sixty-six women. There were no significant differences between the control and intervention groups in terms of gender, age, BMI, or medical, medication or smoking history (Table 1). Similarly, there were no significant differences in $24 \mathrm{~h}$ urinary salt excretion or blood pressure between the groups at baseline (Table 2). No change in $24 \mathrm{~h}$ urinary salt excretion was found in the control group after intervention $(\Delta=-0 \cdot 1$ (SD 3.3$) \mathrm{g} / \mathrm{d}$ ). However, the level was significantly lower in the intervention group $(\Delta=-1.2(\mathrm{sD} 3 \cdot 8) \mathrm{g} / \mathrm{d} ; P=0.039)$, but not significant when the baseline was corrected. Conversely, $24 \mathrm{~h}$ urinary $\mathrm{K}$ excretion was significantly lower after intervention in the control group $(\Delta=-419$ (SD 900) $\mathrm{mg} / \mathrm{d} ; P=0.008$ ); there was no significant difference in the intervention group $(\Delta=-98(\mathrm{sD} 723) \mathrm{mg} / \mathrm{d})$, but a tendency $(P=0.055)$ was found when corrected by baseline values. As a result, the $\mathrm{Na}: \mathrm{K}$ ratio was significantly increased in the control group (baseline, 2.9 (SD 1.4); endline, 3.4 (SD 1.4); $P=0.005$ ), but showed no difference in the intervention group (baseline, 3.4 (SD 1.7); endline, $3 \cdot 1$ (sD 1.6); $P=0 \cdot 289$; Table 2). This difference was still significant when corrected by baseline values $(P=0 \cdot 033)$. 


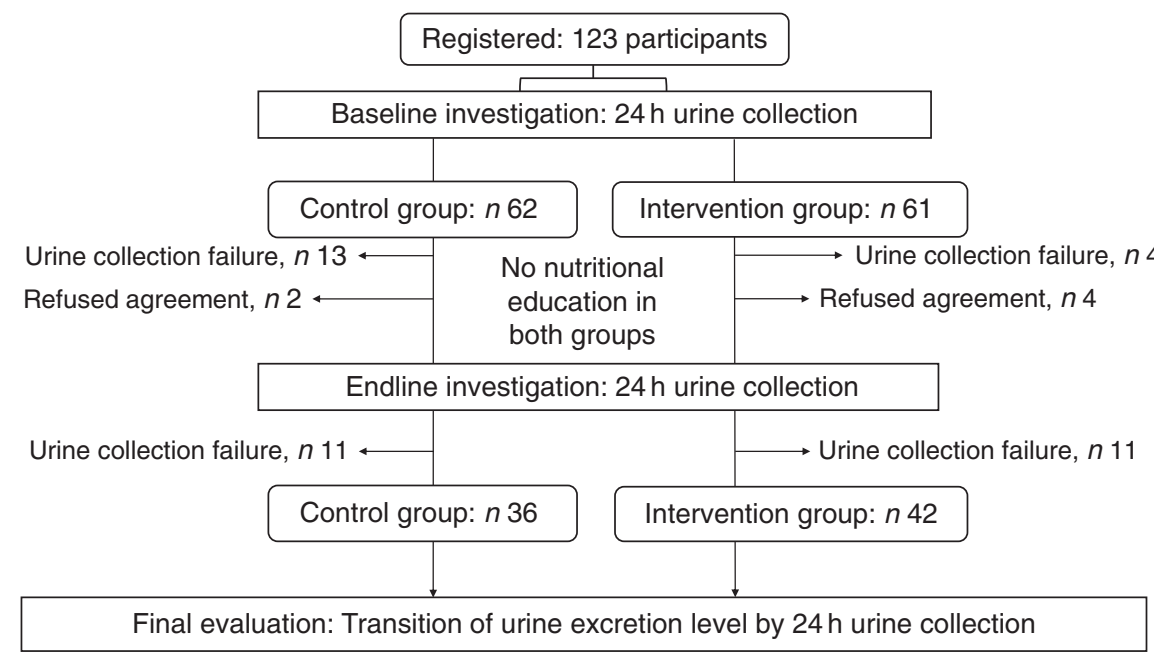

Fig. 2 Allocation of participants. The present study recruited 123 participants and divided them by stratified randomization into a control group with sixty-two individuals and an intervention group with sixty-one individuals. The final analysis involved seventyeight participants (control group, $n$ 36; intervention group, $n$ 42)

Table 1 Characteristics of the study subjects: Japanese adults, 2014

\begin{tabular}{|c|c|c|c|c|}
\hline & All participants & Control group & Intervention group & $P$ value \\
\hline$n$ & 78 & 36 & 42 & \\
\hline \multicolumn{5}{|l|}{ Sex } \\
\hline Male & 12 & 4 & 8 & \\
\hline Female & 66 & 32 & 34 & 0.333 \\
\hline \multicolumn{5}{|c|}{ Age (years) } \\
\hline Mean & $58 \cdot 1$ & $59 \cdot 6$ & $56 \cdot 7$ & \\
\hline SD & $17 \cdot 4$ & 17.5 & 17.5 & 0.468 \\
\hline \multicolumn{5}{|c|}{ BMl $\left(\mathrm{kg} / \mathrm{m}^{2}\right)$} \\
\hline Mean & $22 \cdot 7$ & $22 \cdot 4$ & $23 \cdot 0$ & \\
\hline SD & $3 \cdot 1$ & $2 \cdot 4$ & 3.6 & 0.353 \\
\hline \multicolumn{5}{|c|}{ Hypertension } \\
\hline$n$ & 26 & 13 & 13 & \\
\hline$\%$ & 33.3 & $36 \cdot 1$ & 31.0 & 0.630 \\
\hline \multicolumn{5}{|c|}{ Antihypertensive drugs (other than diuretics) } \\
\hline$n$ & 15 & 8 & 7 & \\
\hline$\%$ & $19 \cdot 2$ & $22 \cdot 2$ & $16 \cdot 7$ & 0.535 \\
\hline \multicolumn{5}{|c|}{ Smoker/past smoker/non-smoker } \\
\hline$n$ & $3 / 9 / 66$ & 1/3/32 & $2 / 6 / 34$ & \\
\hline$\%$ & $3 \cdot 8 / 11 \cdot 5 / 84 \cdot 6$ & $2 \cdot 8 / 8 \cdot 3 / 88 \cdot 9$ & $4 \cdot 8 / 14 \cdot 3 / 81 \cdot 0$ & 0.626 \\
\hline
\end{tabular}

In contrast, there were no significant differences between the two groups in terms of salt excretion, $\mathrm{K}$ excretion, $\mathrm{Na}: \mathrm{K}$ ratio, or systolic and diastolic blood pressure at endline (Table 2). Moreover, BMI did not differ after the intervention in either group (control group: baseline, 22.4

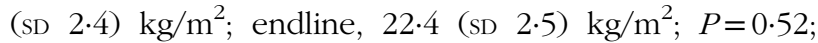
intervention group: baseline, 23.0 (sD 3.6$) \mathrm{kg} / \mathrm{m}^{2}$; endline, $\left.23.0(\mathrm{sD} 3.5) \mathrm{kg} / \mathrm{m}^{2} ; P=0.45\right)$. Similarly, at endline, there was no difference between the two groups in this regard.

Next, we analysed changes in the scores on the salt check sheet after intervention. There was no significant difference in salt intake between the control and intervention groups at baseline, as evaluated using the salt check sheet items and total score. After intervention in the control group, there was no difference in the total 'seasoning/eating-out' score (four items: baseline, 3.1 (SD 2.4); endline, 2.7 (SD 1.9); $P=0.14$ ) or in the total 'all items' score (thirteen items: baseline, $10 \cdot 3$ (SD 4.6); endline, 9.5 (SD 3.9); $P=0.15$ ). However, in the intervention group, both scores were significantly decreased ('seasoning/ eating-out': baseline, 3.2 (SD 2.1); endline, 2.5 (SD 1.9); $P=0.01$; 'all items': baseline, $10 \cdot 6$ (sD 3.8); endline, $9 \cdot 5$ (SD $4 \cdot 2) ; P=0 \cdot 01$ ). Both groups showed a decrease in the 'frequency of high-salt food intake' (seven items) and 'seasoning amount' (two items), but the difference was not significant in either group. In addition, both groups showed a non-significant tendency towards improved salt intake level (Table 3). These results were similar even when participants receiving medication were excluded (data not shown). The distribution of eating behaviour stage showed no difference between the groups at baseline. However, at endline, the intervention group showed a significant increase in eating behaviour stage, while the control group showed no change (Table 3). 
Table 2 Change in $24 \mathrm{~h}$ urine collection and blood pressure variables after 4 weeks of intervention among Japanese adults, 2014

\begin{tabular}{|c|c|c|c|c|c|c|c|c|c|c|}
\hline & \multicolumn{2}{|c|}{ Baseline } & \multicolumn{2}{|c|}{ Endline } & \multicolumn{3}{|c|}{ Change from baseline } & \multicolumn{3}{|c|}{$\begin{array}{c}\text { Adjusted difference } \\
\text { (intervention } v \text {. control) }^{\star}\end{array}$} \\
\hline & Mean & SD & Mean & SD & Mean & $95 \% \mathrm{Cl}$ & $P$ value & Mean & $95 \% \mathrm{Cl}$ & $P$ value \\
\hline \multicolumn{11}{|l|}{ Na excretion (mg/d) } \\
\hline Control group ( $n 36)$ & 3509 & 1501 & 3456 & 1513 & -52 & $-484,379$ & 0.808 & -245 & $-301,791$ & 0.374 \\
\hline Intervention group ( $n$ 42) & \multirow{2}{*}{\multicolumn{2}{|c|}{0.249}} & \multirow{2}{*}{\multicolumn{2}{|c|}{0.954}} & -487 & $-947,-26$ & 0.039 & & & \\
\hline$P$ value & & & & & & & & & & \\
\hline \multicolumn{11}{|l|}{ Salt excretion $(\mathrm{g} / \mathrm{d})$} \\
\hline Control group (n 36) & $9 \cdot 0$ & 3.8 & $8 \cdot 8$ & 3.9 & $-0 \cdot 1$ & $-1 \cdot 2,1 \cdot 0$ & 0.803 & -0.6 & $-2 \cdot 0,0 \cdot 8$ & 0.376 \\
\hline Intervention group ( $n$ 42) & $10 \cdot 0$ & 4.2 & 8.8 & 3.6 & $-1 \cdot 2$ & $-2 \cdot 4,-0.1$ & 0.039 & & & \\
\hline$P$ value & \multicolumn{2}{|c|}{0.252} & \multicolumn{2}{|c|}{0.948} & & & & & & \\
\hline \multicolumn{11}{|l|}{ K excretion (mg/d) } \\
\hline Control group ( $n$ 36) & 2284 & 1058 & 1866 & 706 & -419 & $-723,-114$ & 0.008 & 271 & $-5 \cdot 8,548 \cdot 6$ & 0.055 \\
\hline Intervention group ( $n$ 42) & 2198 & 815 & 2100 & 747 & -98 & $-323,127$ & 0.385 & & & \\
\hline$P$ value & \multicolumn{2}{|c|}{0.683} & \multicolumn{2}{|c|}{0.161} & & & & & & \\
\hline \multicolumn{11}{|l|}{$\mathrm{Na}: \mathrm{K}$ ratio } \\
\hline Control group (n 36) & $2 \cdot 9$ & 1.4 & 3.4 & 1.4 & 0.5 & $0.2,0.8$ & 0.005 & -0.6 & $-1 \cdot 1,0 \cdot 0$ & 0.033 \\
\hline Intervention group ( $n$ 42) & 3.4 & $1 \cdot 7$ & $3 \cdot 1$ & 1.6 & -0.2 & $-0.7,0.2$ & 0.289 & & & \\
\hline$P$ value & \multicolumn{2}{|c|}{0.197} & \multicolumn{2}{|c|}{0.478} & & & & & & \\
\hline \multicolumn{11}{|l|}{ SBP $(\mathrm{mmHg})$} \\
\hline Control group (n 36) & 124.4 & $18 \cdot 3$ & $126 \cdot 4$ & $17 \cdot 6$ & 1.9 & $-1 \cdot 1,5 \cdot 0$ & 0.210 & $-2 \cdot 4$ & $-6 \cdot 7,1.9$ & 0.271 \\
\hline Intervention group ( $n$ 42) & $124 \cdot 3$ & $15 \cdot 6$ & $123 \cdot 9$ & $18 \cdot 1$ & -0.5 & $-3 \cdot 6,2 \cdot 7$ & 0.768 & & & \\
\hline$P$ value & \multicolumn{2}{|c|}{0.887} & \multicolumn{2}{|c|}{0.550} & & & & & & \\
\hline \multicolumn{11}{|l|}{$\mathrm{DBP}(\mathrm{mmHg})$} \\
\hline Control group (n 36) & $74 \cdot 3$ & $10 \cdot 9$ & 73.9 & $9 \cdot 3$ & -0.4 & $-2 \cdot 5,1 \cdot 7$ & 0.720 & 0.4 & $-1 \cdot 9,2 \cdot 7$ & 0.736 \\
\hline Intervention group ( $n$ 42) & $76 \cdot 1$ & $10 \cdot 8$ & $75 \cdot 7$ & $10 \cdot 1$ & -0.4 & $-2 \cdot 0,1 \cdot 2$ & 0.602 & & & \\
\hline$P$ value & \multicolumn{2}{|c|}{0.531} & \multicolumn{2}{|c|}{0.404} & & & & & & \\
\hline
\end{tabular}

SBP, systolic blood pressure; DBP, diastolic blood pressure.

$P$ value compares the mean difference between the baseline and endline changes in the variables between the two groups.

${ }^{*}$ Correction by baseline values using ANCOVA.

Next, we analysed the changes in self-monitored urinary salt excretion after the intervention. The mean ON salt excretion, as measured using the self-monitoring device for ON urine, showed a tendency towards a decrease at 2 , 3 and 4 weeks. However, ANOVA found no significant differences in this regard (Fig. 3(a)). However, the mean ON salt excretion value in weeks 3 and 4 was significantly decreased compared with weeks 1 and 2 (7.7 (SD 1.3) $v$. 7.9 (sD 1.3) g/d; $P=0.02$; Fig. 3(b)). The participants filled their $\mathrm{ON}$ salt excretion values into the self-monitoring graph on a mean of 24.0 (SD 5.1) d, with no difference being found between the groups in this regard.

\section{Discussion}

Salt intake in Japan is considerably higher than in many other countries ${ }^{(6,7)}$ and it is therefore important that researchers and policy makers develop effective saltreduction education. The present study aimed to evaluate the effectiveness of a self-monitoring salt-excretion device as a novel education method. The study was a 4 -week parallel, randomized trial involving two groups.

Interestingly, the two groups in the present study did not receive any nutritional education and only the intervention group, who used the self-monitoring device, showed a significant effect. In a previous report, we found that the use of a self-monitoring device decreased ON salt excretion without any special salt-reduction education.
However, the experiment used no control group ${ }^{(17)}$. In a similar study, Ohta et al. collected $24 \mathrm{~h}$ urine samples from 103 individuals with hypertension ten times over a 5-year period. They showed that feedback regarding urinary salt levels significantly lowered salt excretion levels by $1.4 \mathrm{~g} / \mathrm{d}^{(10)}$. This corroborates the present study, which also found that awareness of one's own urinary salt excretion level significantly affected that level, indicating that the intervention triggered salt-reduction behaviour. Relatedly, eating behaviour stage was improved only in the intervention group, indicating the same conclusion.

The results indicated that the intervention significantly improved the urinary excreted $\mathrm{Na}: \mathrm{K}$ ratio due to tendency of lower salt $\mathrm{Na}$ and equal $\mathrm{K}$ excretion between baseline and endline. There was no such tendency and no significant difference of urinary excreted $\mathrm{Na}: \mathrm{K}$ in the control group. In addition, the scores of the salt check sheet, which evaluates habitual salt intake level ${ }^{(18)}$, showed that the decrease was due to changes in the amount of added seasoning and frequency of eating out. Twenty-four-hour salt and $\mathrm{K}$ excretion showed no significant difference when corrected by baseline values. We believe that evaluation of the educational effect of the self-monitoring device in such a short period either needs usage of urinary excreted $\mathrm{Na}: \mathrm{K}$ ratio as a marker since it is more sensitive; or that a slightly longer period is necessary for detecting improvement of $24 \mathrm{~h}$ urinary salt excretion. However, in the intervention group, the measurements of the self-monitoring salt-excretion device were significantly 
decreased in weeks 3 and $4 v$. weeks 1 and 2, with no difference in BMI. Taken together, these results indicate that information regarding one's own ON salt excretion level, as measured using a self-monitoring device, is effective in reducing salt intake. Studies with larger target populations are necessary to confirm our results.

Numerous studies have found that salt reduction lowers blood pressure ${ }^{(33,34)}$. For instance, Obarzanak et al. conducted a crossover comparison study wherein 188 individuals with hypertension reduced their salt intake from 8.2 to $3.7 \mathrm{~g} / \mathrm{d}$, leading to a significant reduction in mean systolic blood pressure ${ }^{(20)}$. However, in the present study, blood pressure did not change in either group, perhaps because the mean blood pressure of the participants reported herein was within the normal range and because the mean reduction in $24 \mathrm{~h}$ urinary salt excretion in the intervention group was $1.2 \mathrm{~g}$, which, although significant, may have been too small a change to affect blood pressure. However, in a meta-analysis, salt reduction of $1 \mathrm{~g} / \mathrm{d}$ over $>4$ weeks lowered blood pressure by $1 \mathrm{mmHg}$ in hypertensive subjects and by $0.5 \mathrm{mmHg}$ in normotensive subjects ${ }^{(33)}$. In addition, among all patients with hypertension in the USA assumed to take antihypertensive reagents, salt reduction of only $1 \mathrm{~g}$ has a greater prophylactic effect ${ }^{(35)}$. Taken together with these previous reports, the effect of salt reduction in the present paper cannot be overlooked. Future studies must re-evaluate this effect in a large hypertensive group.

After the intervention, the participants in the control group showed a tendency of lower $24 \mathrm{~h}$ urinary K excretion levels and a higher Na:K ratio. However, the intervention group showed no difference. $\mathrm{K}$ counteracts high salt intake-dependent blood pressure elevation ${ }^{(36,37)}$ and higher $\mathrm{Na}: \mathrm{K}$ ratio is related to cardiovascular risk and all-cause mortality rate ${ }^{(38)}$. Thus, the combination of reduced salt excretion and unchanged $\mathrm{K}$ excretion is an important finding. Ideally, reduced salt excretion and increased $\mathrm{K}$ excretion should be observed, but we found this seldom happens simultaneously.

The self-monitoring salt-excretion device cannot estimate urinary $\mathrm{K}$ levels. For this reason, salt-reduction education, $24 \mathrm{~h}$ urine collection, spot-urine tests and second-urine tests after waking up should be conducted to measure $\mathrm{K}$ excretion and $\mathrm{Na}: \mathrm{K}$ ratio ${ }^{(11,12,39)}$. We do not know why urinary $\mathrm{K}$ excretion and resulting $\mathrm{Na} \mathrm{K}$ ratio showed, although not significant, respective lower and higher values in the control group. However, the result implies that it is important to evaluate $\mathrm{K}$ together with $\mathrm{Na}$.

The present study had several limitations. First, in the control group, we found that baseline $24 \mathrm{~h}$ urinary salt excretion level was higher than in the intervention group. Although the significance of this and resulting $24 \mathrm{~h}$ salt excretion became non-significant after correction by baseline values, we need a larger target population to confirm the present study. Nutritional education is generally more effective among individuals with a poor socio-economic 

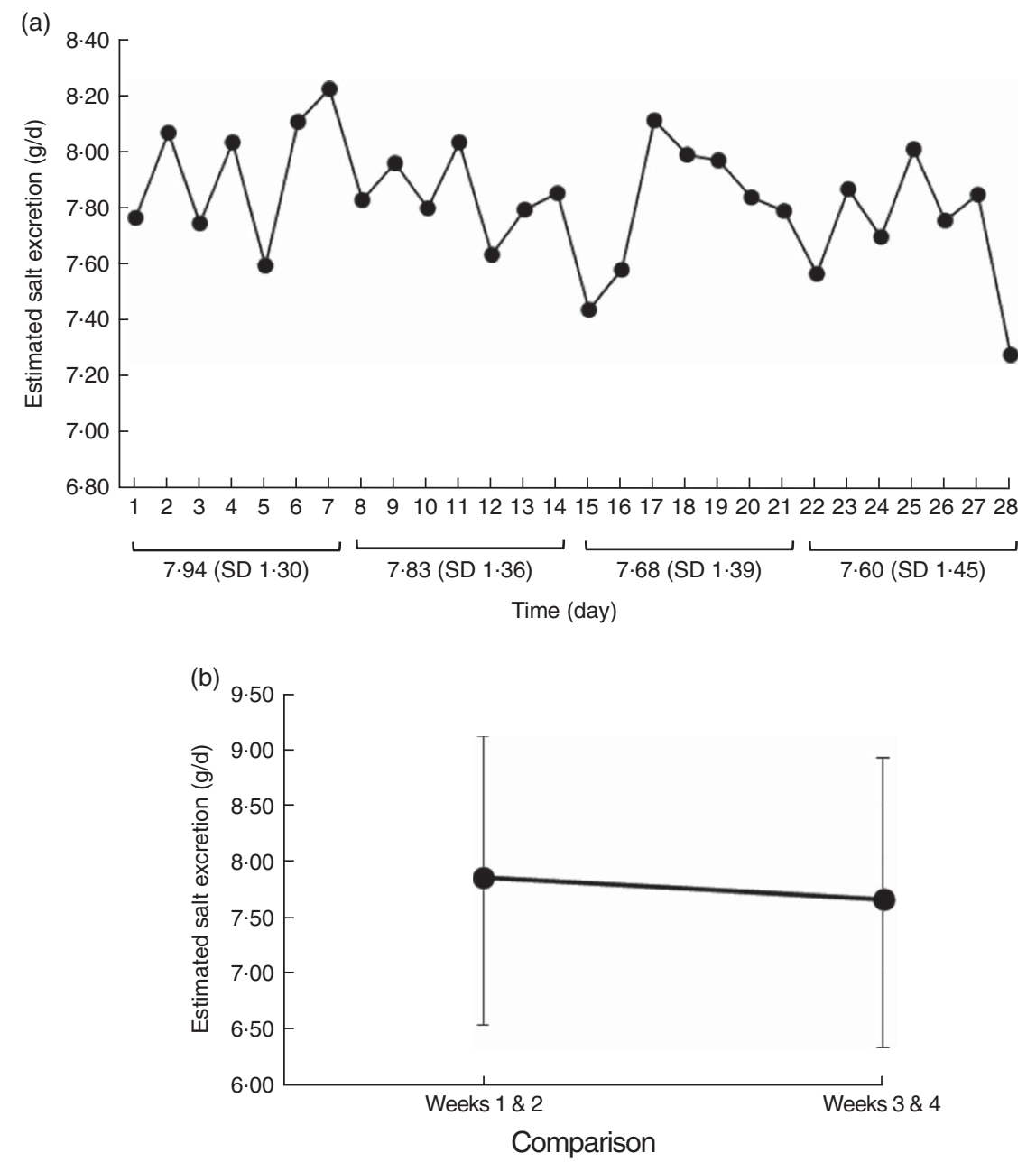

Fig. 3 Changes in estimated salt excretion levels evaluated using overnight urine in the intervention group of forty-two Japanese adults, 2014. (a) The mean estimated overnight salt urine excretion, measured using a self-monitoring device, was lower in weeks 2 and 3 than in week 1. However, it showed no change by multiple comparison. (b) Weeks 3 and 4 showed a significant decrease in mean estimated salt excretion (standard deviations shown by vertical bars) compared with weeks 1 and 2

background $^{(40)}$. Thus, it may be that regression towards the mean had influenced the results. At baseline, we used stratified randomization to ensure that both groups had equal $24 \mathrm{~h}$ salt excretion levels. However, some participants dropped out subsequently because, for example, they failed to collect their $24 \mathrm{~h}$ home urine samples. This may have influenced the results. Indeed, salt excretion, $\mathrm{K}$ excretion and $\mathrm{Na}: \mathrm{K}$ ratio did not differ significantly between the two groups at endline (Table 2), suggesting that the effect of the self-monitoring is limited.

Second, the present study was a short-term one that involved only a few participants with normal mean blood pressure; the resulting salt excretion reduction was small and there was no change in blood pressure. Future studies must involve a longer evaluation period and the use of blood pressure as an outcome in a hypertensive population.

The third limitation was that our salt check sheet and questionnaire about salt-reducing eating behaviour have not been checked for reproducibility. Our future studies will implement a detailed dietary investigation to analyse changes in dietary salt consumption using a self-monitoring device.

The final limitation is that the results of the present study may not be applicable in other groups with different profiles.

\section{Conclusion}

Salt-reduction education using a self-monitoring saltexcretion device helps $24 \mathrm{~h}$ urinary $\mathrm{Na}: \mathrm{K}$ ratio, salt check sheet scores and eating behaviour stage. Thus, the usage of self-monitoring tools has an educational potential in salt intake reduction.

\section{Acknowledgements}

Acknowledgements: The authors wish to thank Ms Masayo Yasumoto, Ms Hiroko Takeuchi, Ayako Irie, Kazuko Ishimoto and Hiromi Misono for their excellent technical assistance, and Dr Hideaki Kumahara for help with statistics. 
Financial support: This work was supported by a Grant-inAid for Scientific Research from the Japan Society for the Promotion of Science (grant number JP25750067). The funder had no role in the design, analysis or writing of this article. Conflict of interest: All authors declare that they have nothing to disclose regarding funding or conflicts of interest with respect to this manuscript. Authorship: Conception, design and overall supervision of study: K.Y. and T.T. Registration of subjects and collection of urine samples: K.Y., E.M., T.K., T.F. and T.I. Analysis and interpretation the data: K.Y., R.M., Y.Mi., Y.Mu., K.O. and M.E. Write up: K.Y., K.O., M.E. and T.I. Ethics of buman subject participation: The study protocol was approved by the ethics committee of Nakamura Gakuen University (Rinir14-001) and adhered to the ethical guidelines of the Declaration of Helsinki. Written informed consent was obtained from the subjects of their own free will. They confirmed that (i) they suffered no disadvantages for refusing the study overview, (ii) they were participating freely, (iii) there were no disadvantages for withdrawing consent after agreeing to participate, (iv) they consented to public dissemination of the results at scientific meetings and in published manuscripts, and (v) their personal information would be protected.

\section{References}

1. Beaglehole R, Bonita R, Horton R et al. (2011) Priority actions for the non-communicable disease crisis. Lancet 377, 1438-1447.

2. Strazzullo P, D'Elia L, Kandala NB et al. (2009) Salt intake, stroke, and cardiovascular disease: meta-analysis of prospective studies. BMJ 339, b4567.

3. Mozaffarian D, Fahimi S, Singh GM et al. (2014) Global sodium consumption and death from cardiovascular causes. $N$ Engl J Med 371, 624-634.

4. Aburto NJ, Ziolkovska A, Hooper L et al. (2013) Effect of lower sodium intake on health: systematic review and metaanalyses. BMJ 346, f1326.

5. Intersalt Cooperative Research Group (1988) Intersalt: an international study of electrolyte excretion and blood pressure. Results for 24 hour urinary sodium and potassium excretion. Intersalt Cooperative Research Group. BMJ 297, 319-328.

6. Anderson CA, Appel LJ, Okuda N et al. (2010) Dietary sources of sodium in China, Japan, the United Kingdom, and the United States, women and men aged 40 to 59 years: the INTERMAP study. $J$ Am Diet Assoc 110, 736-745.

7. Asakura K, Uechi K, Sasaki Y et al. (2014) Estimation of sodium and potassium intakes assessed by two $24 \mathrm{~h}$ urine collections in healthy Japanese adults: a nationwide study. Br J Nutr 112, 1195-1205.

8. He FJ, Brinsden HC \& MacGregor GA (2013) Salt reduction in the United Kingdom: a successful experiment in public health. J Hum Hypertens 28, 345-352.

9. Cohen SJ, Weinberger MH, Fineberg NS et al. (1991) The effect of a household partner and home urine monitoring on adherence to a sodium restricted diet. Soc Sci Med 32, 1057-1061.

10. Ohta Y, Tsuchihashi T, Onaka U et al. (2010) Long-term compliance of salt restriction and blood pressure control status in hypertensive outpatients. Clin Exp Hypertens 32, 234-238.
11. Tanaka T, Okamura T, Miura K et al. (2002) A simple method to estimate populational 24-h urinary sodium and potassium excretion using a casual urine specimen. J Hum Hypertens 16, 97-103.

12. Kawasaki T, Itoh K, Uezono K et al. (1993) Simple method for estimating $24 \mathrm{~h}$ urinary sodium and potassium excretion from second morning voiding urine specimen in adults. Clin Exp Pharmacol Physiol 20, 7-14.

13. Yamasue K, Tochikubo O, Kono E et al. (2006) Selfmonitoring of home blood pressure with estimation of daily salt intake using a new electrical device. J Hum Hypertens 20, 593-598.

14. Yasutake K, Sawano K, Shono N et al. (2013) Validation of a self-monitoring device for estimating 24-hour urinary salt excretion. Asia Pac J Clin Nutr 22, 25-31.

15. Yasutake K, Horita N, Murata Y et al. (2015) Estimated urinary salt excretion by a self-monitoring device is applicable to education of salt restriction. Hypertens Res 38, 143-148.

16. Yasutake K, Horita N, Umeki $\mathrm{Y}$ et al. (2016) Selfmanagement of salt intake: clinical significance of urinary salt excretion estimated using a self-monitoring device. Hypertens Res 39, 127-132.

17. Yasutake K, Sawano K, Yamaguchi S et al. (2011) Selfmonitoring urinary salt excretion in adults: a novel education program for restricting dietary salt intake. Exp Ther Med 2, 615-618.

18. Tsuchihashi T, Masuda K, Oniki H et al. (2013) Assessment of salt intake by using a simple check sheet in hypertensive patients. I Blood Press 20, 1239-1243.

19. Prochaska JO \& Velicer WF (1997) The transtheoretical model of health behavior change. Am J Health Promot 12, 38-48.

20. Obarzanek E, Proschan MA, Vollmer WM et al. (2003) Individual blood pressure responses to changes in salt intake: results from the DASH-Sodium trial. Hypertension 42, 459-467.

21. Fukumoto A, Asakura K, Murakami K et al. (2013) Withinand between-individual variation in energy and nutrient intake in Japanese adults: effect of age and sex differences on group size and number of records required for adequate dietary assessment. J Epidemiol 23, 178-186.

22. Iwahori T, Miura K \& Ueshima H (2017) Time to consider use of the sodium-to-potassium ratio for practical sodium reduction and potassium increase. Nutrients $\mathbf{5}$, E700.

23. Tochikubo O, Uneda S \& Kaneko Y (1983) Simple portable device for sampling a whole day's urine and its application to hypertensive outpatients. Hypertension 5, 270-274.

24. Yasutake K, Miyoshi E, Kajiyama T et al. (2016) Comparison of a salt check sheet with 24-h urinary salt excretion measurement in local residents. Hypertens Res 39, 879-885.

25. Ohta Y, Tsuchihashi T, Fujii K et al. (2004) Improvement of blood pressure control in a hypertension clinic: a 10-year follow-up study. J Hum Hypertens 18, 273-278.

26. Fujimoto K, Sakata T, Etou H et al. (1992) Charting of daily weight pattern reinforces maintenance of weight reduction in moderately obese patients. Am J Med Sci $\mathbf{3 0 3}$, $145-150$.

27. Ministry of Health, Labor and Welfare of Japan (2014) Dietary Reference Intakes for Japanese (in Japanese). Tokyo: Dai-ichi Shuppan Co.

28. Kawano Y, Tsuchihashi T, Matsuura H et al. (2007) Report of the working group for dietary salt reduction of the Japanese Society of Hypertension: (2) Assessment of salt intake in the management of hypertension. Hypertens Res 30, $887-893$.

29. Kobayashi S, Murakami K, Sasaki S et al. (2011) Comparison of relative validity of food group intakes estimated by comprehensive and brief-type self-administered diet history questionnaires against $16 \mathrm{~d}$ dietary records in Japanese adults. Public Health Nutr 14, 1200-1211. 
30. Kobayashi S, Honda S, Murakami K et al. (2012) Both comprehensive and brief self-administered diet history questionnaires satisfactorily rank nutrient intakes in Japanese adults. J Epidemiol 22, 151-159.

31. Sakata S, Tsuchihashi T, Oniki H et al. (2015) Relationship between salt intake as estimated by a brief self-administered diet-history questionnaire (BDHQ) and 24-h urinary salt excretion in hypertensive patients. Hypertens Res 38, 560-563.

32. Tamaki J, Kikuchi Y, Yoshita K et al. (2004) Stages of change for salt intake and urinary salt excretion: baseline results from the High-Risk and Population Strategy for Occupational Health Promotion (HIPOP-OHP) study. Hypertens Res 27, 157-166.

33. He FJ \& MacGregor GA (2002) Effect of modest salt reduction on blood pressure: a meta-analysis of randomized trials. Implications for public health. J Hum Hypertens $\mathbf{1 6}$, 761-770.

34. Dickinson HO, Mason JM, Nicolson DJ et al. (2006) Lifestyle interventions to reduce raised blood pressure: a systematic review of randomized controlled trials. J Hypertens 24, 215-233.
35. Bibbins-Domingo K, Chertow GM, Coxson PG et al. (2010) Projected effect of dietary salt reductions on future cardiovascular disease. $N$ Engl J Med 362, 590-599.

36. Fujita $\mathrm{T} \&$ Ando K (1984) Hemodynamic and endocrine changes associated with potassium supplementation in sodium-loaded hypertensives. Hypertension 6, 184-192.

37. Kawano Y, Minami J, Takishita S et al. (1998) Effects of potassium supplementation on office, home, and 24-h blood pressure in patients with essential hypertension. Am J Hypertens 11, 1141-1146.

38. Yang Q, Liu T, Kuklina EV et al. (2011) Sodium and potassium intake and mortality among US adults: prospective data from the Third National Health and Nutrition Examination Survey. Arch Intern Med 171, 1183-1191.

39. Iwahori T, Ueshima H, Miyagawa $\mathrm{N}$ et al. (2014) Six random specimens of daytime casual urine on different days are sufficient to estimate daily sodium/potassium ratio in comparison to 7-day 24-h urine collections. Hypertens Res 37, 765-771.

40. Denke MA \& Frantz ID Jr (1993) Response to a cholesterollowering diet: efficacy is greater in hypercholesterolemic subjects even after adjustment for regression to the mean. Am J Med 94, 626-631. 